\title{
Exploring the magnetization dynamics of NiFe/Pt multilayers in flexible substrates
}

\author{
M.A. Corrêa ${ }^{\text {a,* }}$, R. Dutra ${ }^{\text {b }}$, T.L. Marcondes ${ }^{b}$, T.J.A. Mori ${ }^{c}$, F. Bohn ${ }^{\text {a }}$, R.L. Sommer ${ }^{\text {b }}$ \\ a Departamento de Física Teórica e Experimental, Universidade Federal do Rio Grande do Norte, 59078-900 Natal, RN, Brazil \\ b Centro Brasileiro de Pesquisas Físicas, Rua Dr. Xavier Sigaud, 150, Urca, 22290-180 Rio de Janeiro, RJ, Brazil \\ ' Laboratório Nacional de Luz Síncrotron, Rua Giuseppe Máximo Scolfaro, 1000, Guará, 13083-100 Campinas, SP, Brazil
}

\section{A R T I C L E I N F O}

\section{Article history:}

Received 30 March 2016

Received in revised form 8 June 2016

Accepted 13 June 2016

Available online 29 June 2016

\section{Keywords:}

Magnetic systems

Magnetization dynamics

Magnetoimpedance effect

Ferromagnetic films

\begin{abstract}
A B S T R A C T
We investigate the structural and magnetic properties, and the magnetization dynamics in $\mathrm{Ni}_{81} \mathrm{Fe}_{19} / \mathrm{Pt}$ multilayer systems grown onto rigid and flexible substrates. The structural characterization shows evidence of a superlattice behavior, while the quasi-static magnetization characterization reveal a weak magnetic anisotropy induced in the multilayers. The magnetization dynamics is investigated through the magnetoimpedance effect. We employ a theoretical approach to describe the experimental magnetoimpedance effect and verify the influence of the effective damping parameter on the magnetization dynamics. Experimental data and theoretical results are in agreement and suggest that the multilayers present high effective damping parameter. Moreover, our experiments raise an interesting issue on the possibility of achieving considerable $\mathrm{MI} \%$ values, even for systems with weak magnetic anisotropy and high damping parameter grown onto flexible substrates.
\end{abstract}

(c) 2016 Elsevier B.V. All rights reserved.

\section{Introduction}

In the recent decades, the magnetization dynamics in ferromagnetic multilayers consisting of a ferromagnetic/metallic nonmagnetic (FM/NM) structure has attracted increasing interest due to the wide potential of application in current and emerging technologies, as well as due to the importance of the understanding in terms of fundamental physics of a richness of phenomena observed in these systems with reduced dimensions [1-7].

Electrical and magnetic properties of the non-magnetic and ferromagnetic layers strongly affect the dynamic magnetic response of a multilayer $[8,9]$. Thus, these properties are keys to set the type of application of a multilayer, since it is based on its whole magnetic behavior. In particular, the magnetic damping plays an important role as a tuning parameter. While systems with low damping parameter are employed in ultrafast sensors [10,11], systems presenting high damping one are explored in spin pumping and inverse spin Hall effect applications [5,12].

Despite the large amount of research reporting on the topic, there are still several aspects on the effective damping parameter $\left(\alpha_{\text {eff }}\right)$ in nanostructures that are not completely understood or can be optimized. Special interest has been devoted to the

\footnotetext{
* Corresponding author.

E-mail address: marciocorrea@dfte.ufrn.br (M.A. Corrêa).
}

magnetization dynamics and control of the relaxation processes which takes place in these systems.

For instance, recently, Ruiz-Calaforra et al. [12] presented a meticulous research on FM/NM multilayers, where FM is a ferromagnetic material, as NiFe and CoFeB, alloys commonly used as free layer in Tunnel Magnetoresistance (TMR) and Giant Magnetostriction (GMS) stacks, while NM is a non-magnetic spacer material, as $\mathrm{Pt}, \mathrm{Al}, \mathrm{Cr}, \mathrm{Ru}$ and $\mathrm{MgO}$, materials frequently employed in spintronics studies, and explored the magnetization dynamics through the ferromagnetic resonance effect (FMR) and inverse spin Hall effect. From the experimental FMR results, an estimative of the effective damping parameter was obtained. As a striking issue, an enhancement of $\alpha_{\text {eff }}$ was verified for the multilayers with Pt as non-magnetic spacer, when compared to the different studied spacers, reaching $\alpha_{\text {eff }} \approx 0.021$ [12].

In addition, for $\mathrm{NiFe} / \mathrm{Pt}$ bilayer wire, Duan et al. [5] reported experimental results of spectral properties of spin-wave modes. From a strict analysis of dynamics properties when the external magnetic field is applied in longitudinal and transversal to the main axis of the wire, an estimative of $\alpha_{\text {eff }}$ is also provided. For this bilayer wire, $\alpha_{\text {eff }} \approx 0.0083$ [5], a lower value noticeably close to the usually verified for ordinary ferromagnetic NiFe thin films.

The aforementioned investigations reveal just a small part of the high complexity of the magnetization dynamics and relaxation processes in nanostructures. Focusing just on films and 


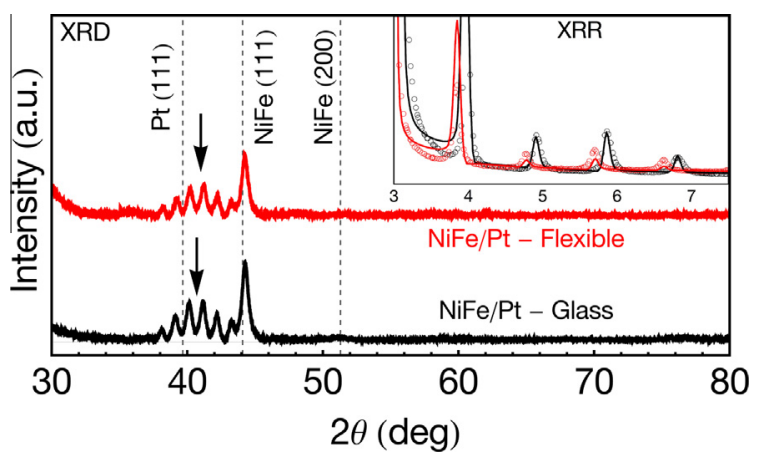

Fig. 1. X-ray diffraction and X-ray reflectometry (inset) for the NiFe/Pt multilayers grown onto rigid and flexible substrates. The dashed lines indicate peaks reported for NiFe (ICDD 01-071-8324) and Pt (ICDD 00-001-1190). The central position of the Pt (111) peak is indicated by the arrows. In the inset, the symbols correspond to the experiment, while the solid lines represent the simulated data for X-ray reflectometry.

multilayers, the effective damping parameter seems to dependent at least on the structure of the sample, deposition technique, parameters used during the growth, as well as on the own materials used as ferromagnetic and non-magnetic layers. The number of variables makes difficult the predictions of $\alpha_{\text {eff }}$ values to nanostructures.

Still, the role of the employed substrate on the effective damping parameter is also not completely clear. Thus, the applicability of multilayers stresses the importance of investigations on the static and dynamic magnetization properties in samples grown onto different substrates. In this way, an increasing number of researches based on multilayers grown onto rigid and flexible substrates has been published, indicating its potential impact in devices applications [7,13-17].

In this work, we report an experimental investigation of the magnetization dynamics through the magnetoimpedance effect in $\mathrm{Ni}_{81} \mathrm{Fe}_{19} / \mathrm{Pt}$ multilayer systems grown onto rigid and flexible substrates. We discuss the experimental results in terms of the mechanisms responsible by the magnetization dynamics and explore, using a theoretical approach, the influence of the effective damping parameter $\alpha_{\text {eff }}$ on the dynamic response. Thus, we show that considerable $\mathrm{MI} \%$ values can be achieved for multilayers grown onto flexible substrates with weak magnetic anisotropy and high damping parameter.

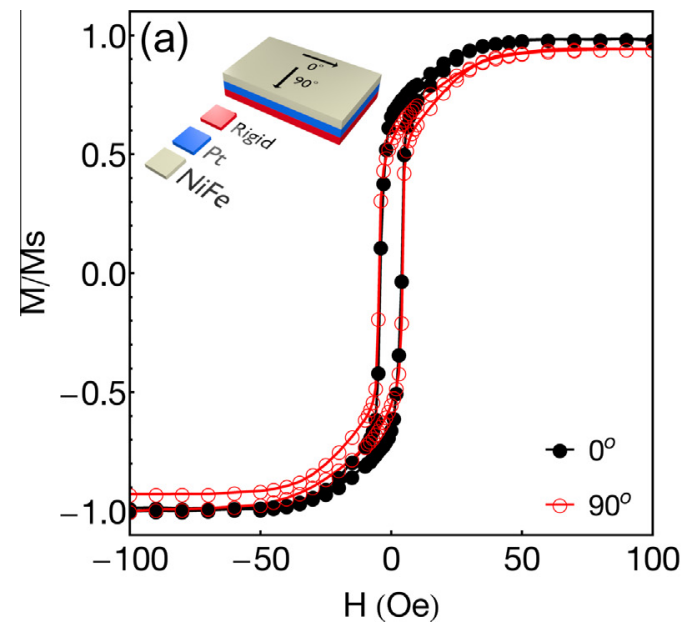

\section{Experiment}

For this study, we produce multilayers with the $\left[\mathrm{Ni}_{81} \mathrm{Fe}_{19}(10-\right.$ $\mathrm{nm}) / \mathrm{Pt}(2 \mathrm{~nm})] \times 30$ structure (from now on NiFe/Pt multilayers), grown onto rigid glass and flexible Kapton ${ }^{\circledR}$ substrates. The samples are deposited by magnetron sputtering, with normal incidence, onto static substrates with dimensions of $10 \times 4 \mathrm{~mm}^{2}$, using the following parameters: base vacuum of $8.0 \times 10^{-8} \mathrm{Torr}$, deposition pressure of 5.0 mTorr with a $99.99 \%$ pure Ar at $50 \mathrm{sccm}$ constant flow, dc source with current of $100 \mathrm{~mA}$ and power of 36 $\mathrm{W}$ for the deposition of the Pt layers, while $65 \mathrm{~W}$ set in the rf power supply for the NiFe layers. Under this conditions, the deposition rates are $2.5 \AA / s$ and $0.74 \AA / \mathrm{s}$.

The chemical modulation of the superstructure and the structural properties are verified by using a Rigaku Miniflex II diffractometer, in the Bragg-Brentano $(\theta-2 \theta)$ geometry, by X-ray reflectometry (XRR) experiments, that probe the quality and uniformity of the layers, as well as by X-ray diffraction (XRD) measurements, which verify the structural character of the films and preferential growth along the out-of-plane direction. The quasistatic magnetic behavior is obtained through magnetization curves measured at room temperature using a Lake Shore model 7404 vibrating sample magnetometer, with maximum in-plane magnetic field of \pm 300 Oe. The curves are acquired along $\left(0^{\circ}\right)$ and perpendicular $\left(90^{\circ}\right)$ the main axis of the multilayer.

Finally, the magnetization dynamics is investigated through the longitudinal magnetoimpedance effect, where magnetic field and current are applied along the direction of the main axis of the multilayer. The measurements are obtained using a spectrumimpedance-network analyzer Agilent model E4991, with E4991A test head connected to a stripline, in which the sample is the central conductor. The curves are taken by acquiring the real $R$ and imaginary $X$ components of the impedance in a wide range of frequencies from $0.5 \mathrm{GHz}$ up to $3.0 \mathrm{GHz}$, with $0 \mathrm{dBm}(1 \mathrm{~mW})$ constant power, and with in-plane magnetic field, varying between \pm 350 Oe, applied along the main axis of the samples.

\section{Results and discussion}

In a first moment, the multilayers are characterized on the structural and quasi-static magnetic point of view.

Fig. 1 shows the XRD and XRR results for the NiFe/Pt multilayers onto rigid and flexible substrates. The predominance of (111) peaks

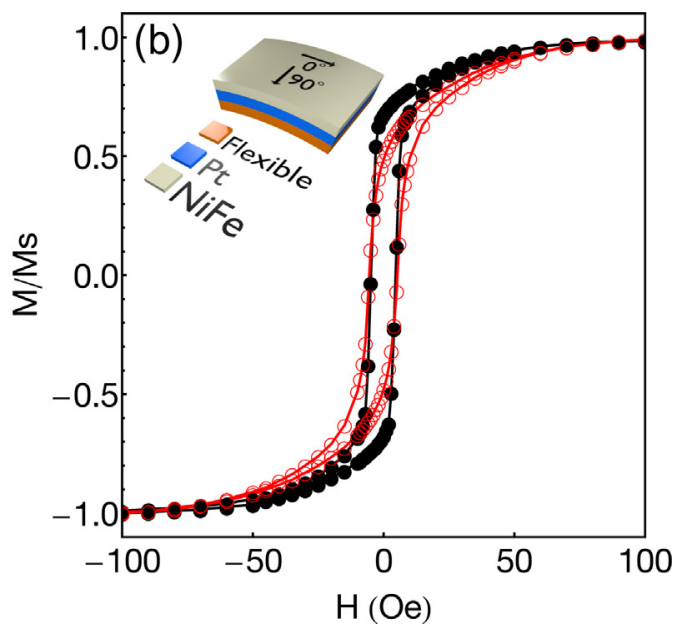

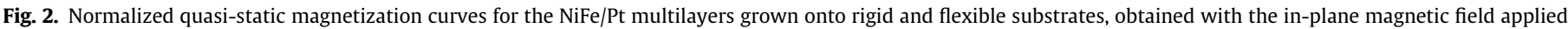
along $\left(0^{\circ}\right)$ and perpendicular $\left(90^{\circ}\right)$ the main axis of the films. 

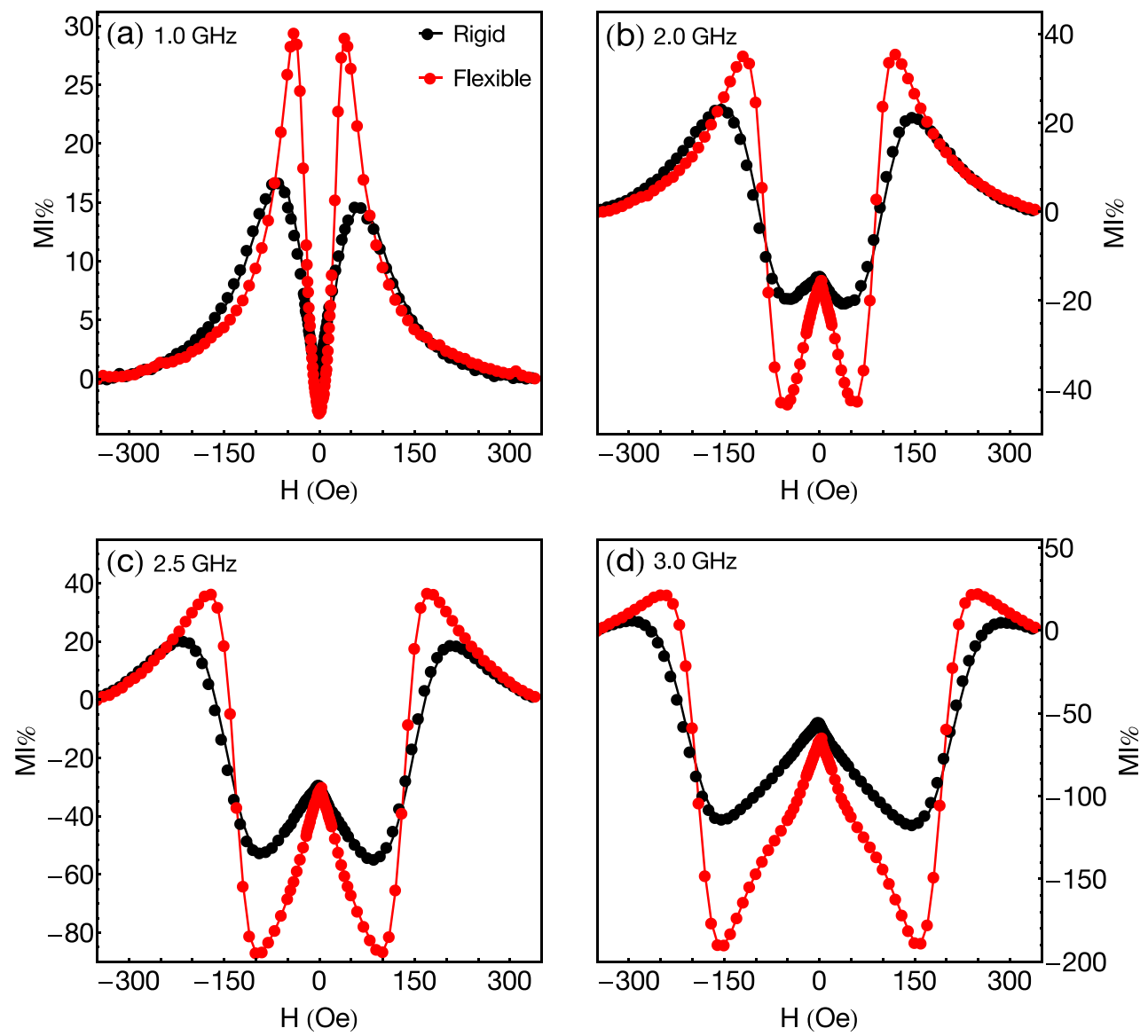

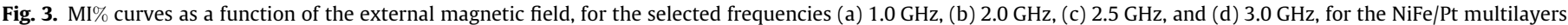
grown onto rigid and flexible substrates.

in the XRD patterns clearly indicate that both $\mathrm{Ni}_{81} \mathrm{Fe}_{19}$ and Pt grow with a preferential mode where the (111) planes have out-of-plane orientation, irrespectively on the employed substrate. The good quality of the superstructure is evidenced by the interference pattern that shows up even at high angles and is enveloped by the Pt (111) peak in the diffractograms. At least 7 interference fringes can be identified with the brightness and resolution of the used diffractometer, and their period corresponds to $9.2 \mathrm{~nm}$ and $9.4 \mathrm{~nm}$ thick superperiods for samples grown on rigid and flexible substrates, respectively. Indeed, crystallite sizes estimated by the DebyeScherrer equation from the full width at half maximum of the (111) peaks always lead to values within the error of the measured layer thickness. These layer thicknesses where checked by simulating the XRR diffratograms, shown in the inset of Fig. 1, and found to be $7.2 \mathrm{~nm}$ and $7.4 \mathrm{~nm}$ for $\mathrm{NiFe}$ in the rigid and flexible samples, respectively, and $2.0 \mathrm{~nm}$ for Pt in both multilayers. The flatness of the multilayers interfaces is also verified by the XRR simulation results and has average values as small as $0.48 \mathrm{~nm}$ and $0.52 \mathrm{~nm}$ for the $\mathrm{NiFe} / \mathrm{Pt}$ interfaces, as well as $0.36 \mathrm{~nm}$ and $0.45 \mathrm{~nm}$ for the $\mathrm{Pt} /$ $\mathrm{NiFe}$ interfaces, in the rigid and flexible multilayers, respectively. Here it is worthwhile to note the slightly higher roughness of the sample grown on the flexible substrate. This sample also presents a stronger tension than the one grown on the rigid substrate. Actually, both multilayers present the Pt layers under tensile stress, stored during deposition, as can be inferred from the shift of the Pt (111) peak, indicated by the arrows, to higher angles with respect to the expected for the bulk state, depicted by the dashed line. The evaluation of the strain from the $d_{111}$ values leads to approximately -0.0251 for the rigid sample and -0.0291 for the flexible one.
Fig. 2 shows the quasi-static magnetization curves, measured with the in-plane magnetic field applied both along and perpendicular to the main axis of the NiFe/Pt multilayers grown onto rigid and flexible substrates. The angular dependence of the magnetization curves indicates a weak magnetic anisotropy, induced by the stray field of the gun of the sputtering system used for the multilayers deposition. Both multilayers share the magnetic behavior, as expected since they present very similar structural properties. From the measurements along of the main axis, with saturation field $H_{s} \approx 50 \mathrm{Oe}$, and coercive fields $H_{c}$ of 4.3 Oe and 5.1 Oe for multilayers grown onto rigid and flexible substrates, respectively. The soft magnetic properties are expected since they are commonly verified for magnetic multilayers in which the ferromagnetic material is $\mathrm{Ni}_{81} \mathrm{Fe}_{19}$. At the same time, a striking issue resides in the evident reproducibility of the quasi-static magnetic properties, irrespective on the substrate.

Regarding the magnetization dynamics and magnetoimpedance effect, to make clearer the comparision of the results, traditionally, the MI ratio is defined as

$\mathrm{MI} \%=\frac{Z(H)-Z\left(H_{\max }\right)}{Z\left(H_{\max }\right)} \times 100$,

where $Z(H)$ is the impedance value for a given external magnetic field value and $Z\left(H_{\max }\right)$ the electrical impedance for the maximum applied external magnetic field, where the sample is magnetically saturated.

Fig. 3 shows the MI\% as a function of the external magnetic field, for selected frequencies, for both NiFe/Pt multilayers. Given that the multilayers in distinct substrates present similar structural character and quasi-static magnetic properties, the 
differences of amplitudes noticed in the MI\% curves may be associated to the distinct electrical and mechanical properties of the own substrates. Despite this, the very same peaks structure is verified irrespective of the employed substrate.

At moderate frequencies, up to $\sim 0.85 \mathrm{GHz}$, not shown here, the impedance peaks are close to the coercive field, $\sim 5$ Oe. This feature reflects the fact that the skin effect is the main responsible for the MI variations [18]. Beyond $\sim 0.85 \mathrm{GHz}$, the displacement of the peaks position toward higher magnetic field values suggests that the strong skin effect and the FMR effect become the main mechanisms responsible for the MI variations. Moreover, the curves evolve from double peak structure, at $1.0 \mathrm{GHz}$, to a multiple peak structure, for frequencies above $1.5 \mathrm{GHz}$. In particular, the central peak, located around zero magnetic field, is a consequence of the
The influence of $\alpha_{\text {eff }}$ on the MI effect, as well as on the behavior of the transverse magnetic permeability and impedance, in a multilayered system can be theoretically investigated by considering an approach recently employed by our group [18]. This approach takes into account the appropriate magnetic free energy density for the investigated structure, and a general magnetic susceptibility model proposed by Spinu et al. [20], which describes its dependence with both frequency and magnetic field. From here, it is possible to obtain the transverse magnetic permeability for planar geometry from the susceptibility and, in turn, describe the MI behavior by using different models [21,22], according to the system structure, for a wide range of frequencies and external magnetic field. In this case, the transverse magnetic permeability can be written as [18]

$\mu_{x x}=1+4 \pi \kappa \sin ^{2} \varphi_{M} \times\left[\left(\omega_{r}^{2}-\omega^{2}\right)\left(1+\alpha^{2}\right) \gamma \xi_{\theta \theta}+\alpha M_{s} \omega^{2} \Delta \omega+i\left[-\left(1+\alpha^{2}\right) \gamma \omega \Delta \omega \xi_{\theta \theta}+\alpha M_{s} \omega\left(\omega_{r}^{2}-\omega^{2}\right)\right]\right]$,

higher contribution of the imaginary part of the impedance as the frequency increases [18].

The emergence of the FMR effect can be also confirmed by considering the plot of the imaginary component as a function of the real component of the impedance [19]. This plot provides evidence of a phase shift between the voltage and current that is dependent on the applied field. In this case, a circular trajectory indicates a strong contribution of the FMR effect to the MI changes [19].

Fig. 4 shows the behavior of $X$ as a function of $R$ for selected frequencies over the entire range of applied magnetic field for the studied multilayers. Below $\sim 0.85 \mathrm{GHz}$, not shown here, the FMR contribution is not observed and the circular behavior is not evident. Above $\sim 0.85 \mathrm{GHz}$, irrespective on the substrate, the shape of the presented curves, as a circular trajectory, clearly infers the FMR effect as the main mechanism responsible for the MI variations at this frequency range, in concordance with the previously discussed.

Our experiments raise an interesting issue on the possibility of achieving considerable MI\% values for multilayers with weak magnetic anisotropy and expected high effective damping parameter $\alpha_{\text {eff }}$. As aforementioned, NiFe/Pt multilayers present an enhancement of $\alpha_{\text {eff }}$ [12], when compared to multilayers composed by distinct non-magnetic spacer materials. Consequently, the MI curves may be strongly affected, a fact mainly related with the MI peaks intensity and the peaks position in magnetic field.

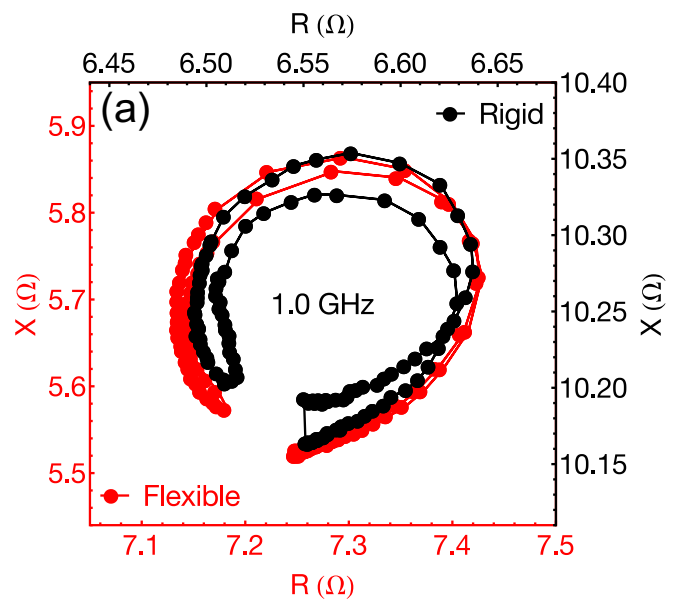

where $\omega_{r}$ and $\Delta \omega$ are known, respectively, as the resonance frequency and width of the resonance absorption line from the FMR theory. At the same time, $\xi_{\theta \theta}$ is the second derivatives of the magnetic free energy density at an equilibrium position, defined by the magnetization vector, as described in Ref. [18]. Finaly, $\gamma$ and $\alpha$ are the giromagnetic factor and the damping parameter, respectively. Here, we consider $\alpha=\alpha_{\text {eff }}$.

The multilayers consists of $N$ ferromagnetic layers separated by metallic non-magnetic layers. To model it, a modified StonerWohlfarth model can be considered and the magnetic free energy density can be written as

$$
\xi=\sum_{i=1}^{N}\left[-\vec{M}_{i} \cdot \vec{H}-\frac{H_{k i}}{2 M_{s i}}\left(\vec{M}_{i} \cdot \hat{u}_{k i}\right)^{2}+4 \pi M_{s i}^{2}\left(\widehat{M}_{i} \cdot \hat{n}\right)\right]
$$

where $\hat{n}$ is a versor oriented perpendicularly to the film plane, $N$ is the number of ferromagnetic layers, $\vec{M}_{i}$ and $M_{s i}$ are the magnetization vector and saturation magnetization for each ferromagnetic layer, respectively, $H_{k i}=2 K_{u i} / M_{s i}$ is the anisotropy field for each layer, and $K_{u i}$ is the uniaxial anisotropy constant, directed along $\hat{u}_{k i}$, for each layer. In a traditional multilayered system, it is reasonable to assume $M_{s 1}=M_{s 2}=\cdots=M_{s N}=M_{s}, K_{u 1}=K_{u 2}=\cdots=K_{u N}=$ $K_{u}, \hat{u}_{k 1}=u_{k 2}=\cdots=\hat{u}_{k N}=\hat{u}_{k}$, since the layers are made of similar ferromagnets.

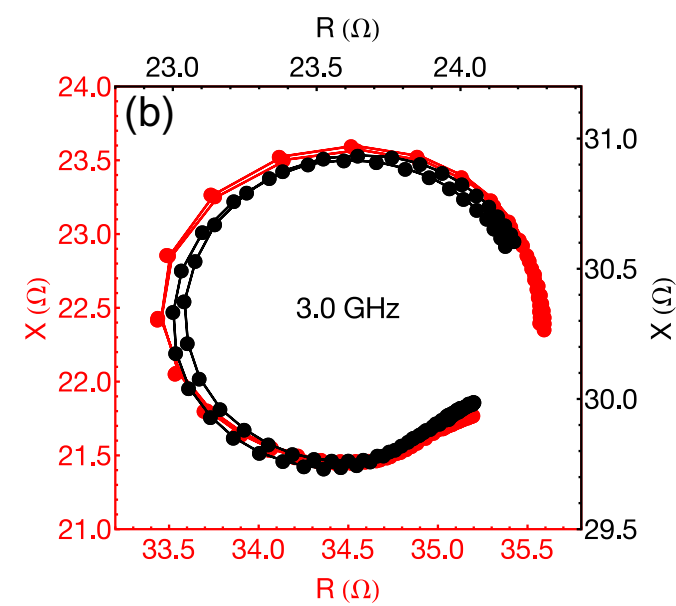

Fig. 4. Real $R$ component as a function of the imaginary $X$ component of the impedance for the selected frequencies (a) $1.0 \mathrm{GHz}$ and (b) $3.0 \mathrm{GHz}$. 

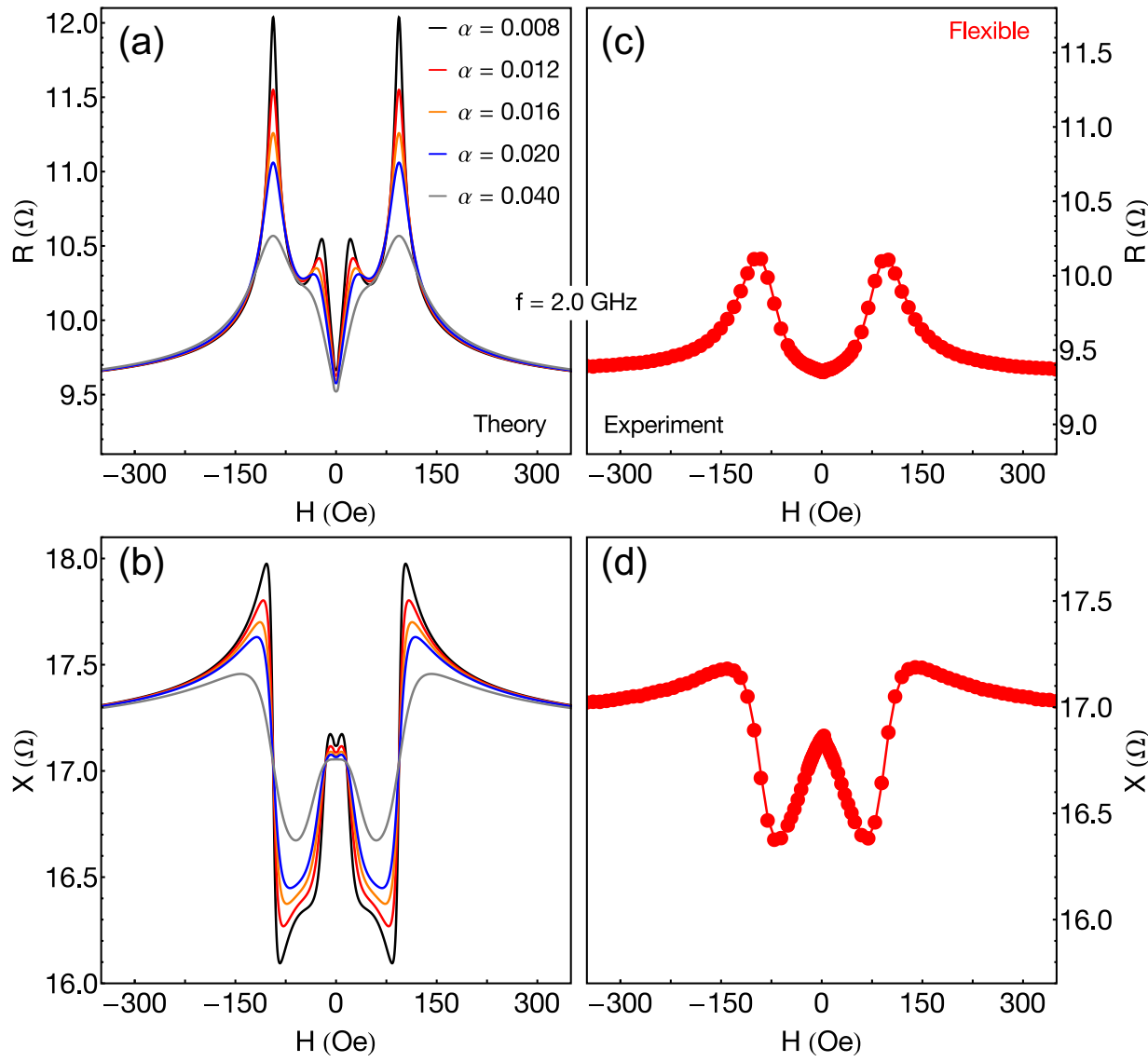

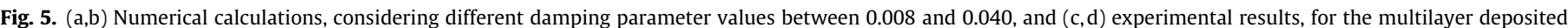

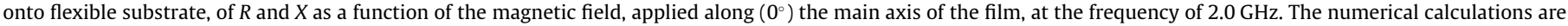

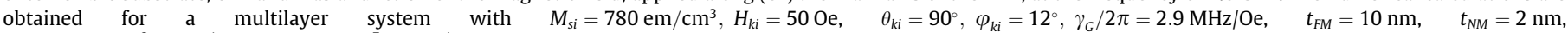

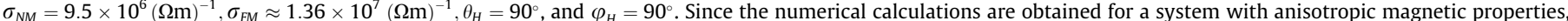

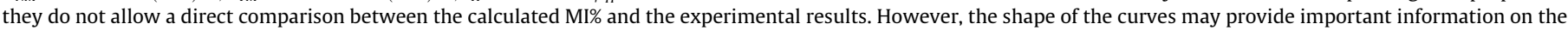

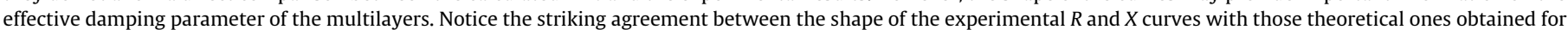
$\alpha \approx 0.040$.

The magnetoimpedance behavior in a multilayered system can be mimic through the model for a trilayered system reported by Panina et al. [18,22], where

$\frac{Z}{R_{d c}}=\left(\eta_{m} \eta_{f}\right)\left[\frac{\operatorname{coth}\left(\frac{\eta_{m} \sigma_{F M}}{\mu \sigma_{N M}}\right) \operatorname{coth}\left(\eta_{f}\right)+\frac{2 \eta_{m}}{k_{N M} t_{N M}}}{\operatorname{coth}\left(\frac{\eta_{m} \sigma_{F M}}{\mu \sigma_{N M}}\right)+\frac{2 \eta_{m}}{k_{N M} t_{N M}} \operatorname{coth}\left(\eta_{f}\right)}\right]$,

where $\mu$ is the magnetic permeability for the ferromagnetic layers, which in our case is $\mu=\mu_{x x}, \sigma_{F M}$ and $\sigma_{N M}$ are the conductivities of the ferromagnetic and non-magnetic layers, respectively, and

$\eta_{m}=\frac{k_{N M} t_{N M}}{2}\left(\frac{\mu \sigma_{N M}}{\sigma_{F M}}\right), \quad \eta_{f}=k_{F M} t_{F M}$,

$k_{N M}=\frac{(1-i)}{\delta_{N M}}, \quad k_{F M}=\frac{(1-i)}{\delta_{F M}}$,

$\delta_{N M}=\left(2 \pi \sigma_{N M} \omega\right)^{-1 / 2}, \quad \delta_{F M}=\left(2 \pi \sigma_{F M} \omega \mu\right)^{-1 / 2}$,

with $\omega$ as the angular frequency.

To perform the numerical calculation, using the same configuration and parameters designations employed in Ref. [18], we consider a theoretical system with an uniaxial anisotropy described by: $M_{s i}=780 \mathrm{em} / \mathrm{cm}^{3}, H_{k i}=50$ Oe, $\theta_{k i}=90^{\circ}, \varphi_{k i}=12^{\circ}, \gamma_{G} / 2 \pi=$ $2.9 \mathrm{MHz} / \mathrm{Oe}, t_{F M}=10 \mathrm{~nm}, t_{N M}=2 \mathrm{~nm}, \theta_{H}=90^{\circ}$, and $\varphi_{H}=90^{\circ}$. In particular, the thickness of the metallic non-magnetic layer is thick enough to neglect the bilinear and biquadratic coupling between the ferromagnetic layers [18,23]. Moreover, we employ $\sigma_{N M}=9.5 \times 10^{6}(\Omega \mathrm{m})^{-1}$ for the Pt layers, and $\sigma_{F M} \approx 1.36 \times$ $10^{7}(\Omega \mathrm{m})^{-1}$ for NiFe layers.

The electrical contribution to the MI of the multilayer is obtained fitting the measured $R$ and $X$ curves as a function of the frequency for the highest magnetic field value, where the sample is magnetically saturated, the same procedure used in the ref. [18]. The parameters are fixed to describe the MI behavior. Thus, the real $R$ and imaginary $X$ components of the longitudinal impedance as a function of field and frequency for multilayered system can be calculated.

In a similar way to the theoretical study reported in Ref. [18], Fig. 5 shows numerical calculations, considering different damping parameter values between 0.008 and 0.040 , and experimental data, for the multilayer deposited onto flexible substrate, of $R$ and $X$ as a function of field for a selected frequency value. Moreover, Fig. 6 shows numerical calculations of the $R$ and $X$, considering different damping parameter values, as a function of the frequency for distinct magnetic field values.

From both, the numerical calculations clearly evidence the strong dependence of the impedance components, and consequently the own impedance, with the damping parameter. The magnitude of the damping parameter directly influences the shape of the curves. It can easily be noted that an increase of the damping parameter leads to a significative reduction of the $R$ and $X$ 

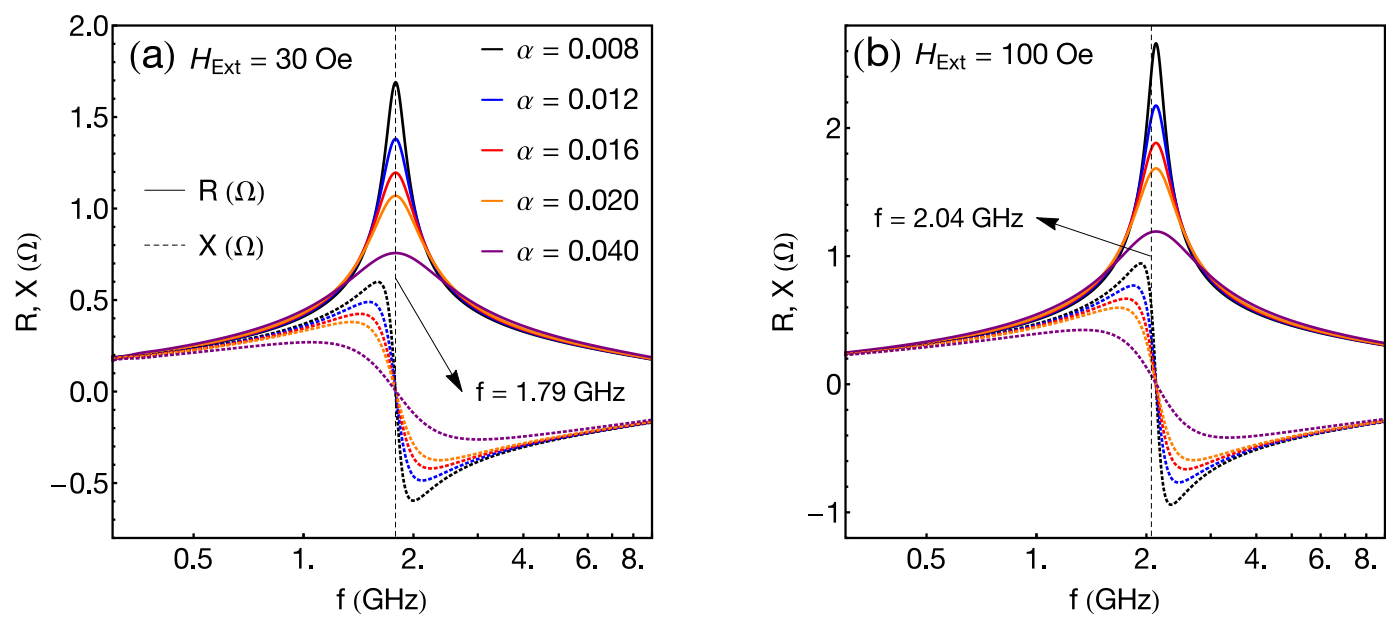

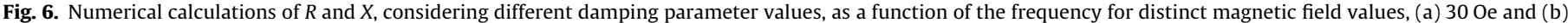
100 Oe. The calculations are performed using parameters similar to those previously employed.

variations, resulting in broader peaks with smaller amplitudes for both components. Moreover, a displacement of the $X$ peak position in the magnetic field is observed when different $\alpha$ values are considered, as verified in Fig. 5(a,b).

However, strikingly, as evidenced in Fig. 6, in both saturated and unsaturated states, i.e. different field values, the peak position of the real component, and the simultaneous zero crossing of the imaginary one, is located at the same frequency position, irrespectively on the damping parameter values, suggesting that the FMR frequency for a given external magnetic field is not influenced by this parameter.

Despite the numerical calculations are obtained for a system with anisotropic magnetic properties and, for this reason, they do not allow a direct comparison between the calculated MI\% and the presented experimental results, the shape of the curves may provide important information on the effective damping parameter of the multilayers. Notice the striking agreement between the shape of the experimental $R$ and $X$ curves with those theoretical ones obtained for $\alpha \approx 0.040$. In this sense, although the precise estimative for $\alpha_{\text {eff }}$ is a hard task and out of the scope of the present work, it is reasonable to interpret this agreement as an indication that the effective damping parameter for the investigated $\mathrm{NiFe} / \mathrm{Pt}$ multilayers has the same order of magnitude, presenting a value within the range between 0.030 and 0.050 , in concordance with the results previously reported for similar system [12]. In particular, we understand that the high damping parameter inferred for the multilayers is a consequence of the use of the Pt as a nonmagnetic spacer material.

Thus, this analysis of the magnetization dynamics opens new possibilities to obtain multilayers with considerable MI variations, linking structures with high damping parameter constant, and soft magnetic properties.

\section{Conclusion}

In conclusion, we have investigated the structural and magnetic properties and the magnetization dynamics, through the magnetoimpedance effect, in $\mathrm{Ni}_{81} \mathrm{Fe}_{19} / \mathrm{Pt}$ multilayers grown onto rigid and flexible substrates. We have verified that these multilayers present evidence of a superlattice behavior and weak magnetic anisotropy. From the dynamic magnetic characterization, we have observed that the magnetoimpedance curves can be explained in terms of the mechanisms responsible by the magnetization dynamics at different frequency ranges, in concordance with the expected for magnetic systems with reduced dimensions at high frequencies. We have employed a theoretical approach to describe the magnetoimpedance effect and verified the influence of the effective damping parameter on the magnetization dynamics. In particular, although the precise estimative for $\alpha_{\text {eff }}$ is not allowed, the comparison between the shape of the experimental data and theoretical results suggests that the NiFe/Pt multilayers have high damping parameter constant, presenting a value within the range between 0.030 and 0.050 . The experiments shows that multilayers grown onto flexible substrates which have weak magnetic anisotropy and high effective damping parameter $\alpha_{\text {eff }}$ may achieve considerable MI\% values.

\section{Acknowledgments}

The research is supported by the Brazilian agencies CNPq (Grants 306423/2014-6, 471302/2013-9, 306362/2014-7, and 441760/2014-7), CAPES, and FAPERN (Pronem 03/2012).

\section{References}

[1] Y. Tserkovnyak, A. Brataas, G.E.W. Bauer, Phys. Rev. B 66 (2002) 224403.

[2] A. Rajanikanth, S. Kasai, N. Ohshima, K. Hono, App. Phys. Lett. 97 (2010) 022505.

[3] W. Lin et al., Thin Solid Films 533 (2013) 70.

[4] A.A. Baker et al. J. Appl. Phys. 115 (2014) 17D140.

[5] Z. Duan, C.T. Boone, X. Cheng, I.N. Krivorotov, Phys. Rev. B 90 (2014) 024427.

[6] E. Fernandez, G.V. Kurlyandskaya, A. Garcia-Arribas, A.V. Svalov, Nanosacale Res. Lett. 7 (2012) 230.

[7] B. Li, M.N. Kavaldzhiev, J. Kosel, J. Magn. Magn. Mater. 378 (2015) 499.

[8] M. Melzer et al., Nano Lett. 11 (2011) 2522.

[9] M. Gueye et al., J. Phys. D: Appl. Phys. 49 (2016) 145003.

[10] A.M. Silva et al., Mat. Let. 156 (2015) 90.

[11] M. Belmeguenai et al., J. Appl. Phys. 115 (2014) 043918.

[12] A. Ruiz-Calaforra et al., J. Appl. Phys. 117 (2015) 163901.

[13] Y. Cui et al., Appl. Phys. Lett. 102 (2013) 162403.

[14] B. Belmeguenai et al., Phys. Rev. B 87 (2013) 184431.

[15] S. Dokupil, M.T. Bootsmann, S. Stein, M. Löhndorf, E. Quandt, J. Magn. Magn. Mater. 290 (2005) 795.

[16] T. Uhrmann et al., J. Magn. Magn. Mater. 307 (2006) 209.

[17] W. Karboul-Trojet, Y. Roussigné, D. Faurie, S.M. Chérif, Eur. Phys. J. B 85 (2012) 339.

[18] M.A. Corrêa, F. Bohn, R.B. da Silva, R.L. Sommer, J. Appl. Phys. 116 (2014) 243904.

[19] J.M. Barandiaran, A. Garcia-Arribas, D. de Cos, J. Appl. Phys. 99 (2006) 103904

[20] L. Spinu, I. Dumitru, A. Stancu, D. Cimpoesu, J. Magn. Magn. Mater. 296 (2006) 1.

[21] L. Kraus, J. Magn. Magn. Mater. 195 (1999) 764.

[22] L. Panina, K. Mohri, Sens. Act. A. 81 (2000) 71.

[23] S.S.P. Parkin, N. More, K.P. Roche, Phys. Rev. Lett. 64 (1990) 2304. 